\title{
LOCAL EXISTENCE AND BLOW UP OF SOLUTIONS FOR A COUPLED VISCOELASTIC KIRCHHOFF-TYPE EQUATION WITH DEGENERATE DAMPING
}

\section{ERHAN PIŞKIN AND FATMA EKINCI}

Received 07 September, 2020

Abstract. In this paper, we consider the initial boundary value problem of a coupled viscoet
Kirchhoff-type equations with degenerate damping:
\[ \left\{\begin{array}{l}u_{t t}-M\left(\|\nabla u\|^{2}\right) \Delta u+\int_{0}^{t} \mu_{1}(t-s) \Delta u(s) d s+\left(|u|^{k}+|v|^{l}\right)\left|u_{t}\right|^{p-1} u_{t}=f_{1}(u, v), \\ v_{t t}-M\left(\|\nabla v\|^{2}\right) \Delta v+\int_{0}^{t} \mu_{2}(t-s) \Delta v(s) d s+\left(|v|^{\theta}+|u|^{\rho}\right)\left|v_{t}\right|^{q-1} v_{t}=f_{2}(u, v) .\end{array}\right. \]

Firstly, we prove a local existence theorem by using the Faedo-Galerkin approximations. Then, we study blow up of solutions when initial energy is positive.

2010 Mathematics Subject Classification: 35B40; 35B44; 35L05; 35L75

Keywords: Kirchhoff type equation, local existence, blow up, viscoelastic equation, degenerate damping, energy

\section{INTRODUCTION AND PRELIMINARIES}

This paper is concerned with the local existence and blow up of solutions to the following viscoelastic Kirchhoff-type equation with degenerate damping:

$$
\left\{\begin{array}{cc}
u_{t t}-M\left(\|\nabla u\|^{2}\right) \Delta u+\int_{0}^{t} \mu_{1}(t-s) \Delta u(s) d s+\left(|u|^{k}+|v|^{l}\right)\left|u_{t}\right|^{p-1} u_{t}=f_{1}(u, v), & (x, t) \in \Omega \times(0, T), \\
v_{t t}-M\left(\|\nabla v\|^{2}\right) \Delta v+\int_{0}^{t} \mu_{2}(t-s) \Delta v(s) d s+\left(|v|^{\theta}+|u|^{\rho}\right)\left|v_{t}\right|^{q-1} v_{t}=f_{2}(u, v), & (x, t) \in \Omega \times(0, T), \\
u(x, t)=v(x, t)=0, & (x, t) \in \partial \Omega \times(0, T), \\
u(x, 0)=u_{0}(x), u_{t}(x, 0)=u_{1}(x), & x \in \Omega, \\
v(x, 0)=v_{0}(x), v_{t}(x, 0)=v_{1}(x), & x \in \Omega,
\end{array}\right.
$$

where $\Omega$ is a bounded domain of $R^{n}(n \geq 1)$ with smooth boundary $\partial \Omega, p, q \geq 1$, $j, k, l, \theta, \rho \geq 0 ; \mu_{i}():. R^{+} \rightarrow R^{+}, f_{i}(.,):. R^{2} \longrightarrow R(i=1,2)$ are given functions to

The authors express their sincere thanks to DUBAP (ZGEF.19.010) for its support. 
be specified later. $M(s)$ is a nonnegative $C^{1}$ function satisfying

$$
M(s)=b_{1}+b_{2} s^{\gamma},
$$

where $\gamma, s \geq 0$ and $b_{1}=b_{2}=1$.

For last years, the study on nonlinear evolution equations with damping terms has been an interesting topic. These problems have wide range of applications in the context of fluid flows, viscosity effects often arise as damping terms in evolution equations. In addition, in the theory of classical mechanics, the physical problems of vibrating membranes, strings or shells in elastic media, damping terms reflect the internal energy that is dissipated by the motion. There is an extensive literature on this kind of problems having damping terms. For instance $[4,11,12,15]$ and one of them is our work [9] where we investigated problem (1.1) and obtained global existence and the general decay of the global solution. Then we proved blow up of solutions of non-linear wave equations having negative initial energy.

We now state other related problem in the literature. Firstly, we mention the pioneer work of $\mathrm{Wu}$ [17], where he studied a general decay of the solution for the following famous system

$$
\left\{\begin{array}{l}
u_{t t}-\Delta u+\int_{0}^{t} \mu_{1}(t-s) \Delta u(s) d s+\left(|u|^{k}+|v|^{l}\right)\left|u_{t}\right|^{p-1} u_{t}=f_{1}(u, v), \\
v_{t t}-\Delta v+\int_{0}^{t} \mu_{2}(t-s) \Delta v(s) d s+\left(|v|^{\theta}+|u|^{\rho}\right)\left|v_{t}\right|^{q-1} v_{t}=f_{2}(u, v) .
\end{array}\right.
$$

Then, Pişkin et al. [10] studied local existence and uniqueness results by using the Faedo-Galerkin method with a new scenario under appropriate assumptions on degenerate damping terms, the parameters and the relaxation functions $w_{i},(i=1,2)$. In addition, some author studied blow up, existence and decay of the solutions (1.2) for $k=l=\theta=\rho=0($ see $[2,3,6-8,14])$.

Rammaha and Sakuntasathien [13] and Zennir et al. [1, 18] investigated the following famous system with degenerate damping

$$
\left\{\begin{array}{l}
u_{t t}-\Delta u+\left(|u|^{k}+|v|^{l}\right)\left|u_{t}\right|^{p-1} u_{t}=f_{1}(u, v), \\
v_{t t}-\Delta v+\left(|v|^{\theta}+|u|^{\rho}\right)\left|v_{t}\right|^{q-1} v_{t}=f_{2}(u, v) .
\end{array}\right.
$$

The authors considered well posedness of solutions, blow up and growth properties.

Moreover, Liu et al. [5] investigated the following system with weak damping

$$
\left\{\begin{array}{c}
u_{t t}-M\left(\|\nabla u\|^{2}\right) \Delta u+\int_{0}^{t} \mu_{1}(t-s) \Delta u(s) d s-\Delta u_{t}=f_{1}(u, v), \\
v_{t t}-M\left(\|\nabla v\|^{2}\right) \Delta v+\int_{0}^{t} \mu_{2}(t-s) \Delta v(s) d s-\Delta v_{t}=f_{2}(u, v) .
\end{array}\right.
$$

Under some conditions, they proved blow up and general decay result.

The content of this paper is organized as follows: In Section 1, we give necessary assumptions and notation that will be used later. In Section 2, firstly, we give definition of weak solution then we investigate the local existence of weak solutions by Galerkin's approximation. In Section 3, we obtain finite time blow up of solutions with positive initial energy. 
Throughout this paper, we denote the standard $L^{2}(\Omega)$-norm by $\|\cdot\|=\|\cdot\|_{L^{2}(\Omega)}$ and $L^{p}(\Omega)$-norm by $\|\cdot\|=\|\cdot\|_{L^{p}(\Omega)}$.

We need the following assumptions to state and prove our results.

(A1) $\mu_{i}(i=1,2)$ are nonincreasing relaxation functions and satisfy

$$
\mu_{i}(s) \geq 0, \mu_{i}^{\prime}(s) \leq 0, \quad 1-\int_{0}^{\infty} \mu_{i}(s) d s=l_{i}>0, s \geq 0 .
$$

(A2) For the nonlinearity in damping, we suppose that

$$
\left\{\begin{array}{c}
1 \leq p, q \text { if } n=1,2 \\
1 \leq p, q \leq \frac{n+2}{n-2} \text { if } n \geq 3 .
\end{array}\right.
$$

We pick up $f_{1}(u, v)$ and $f_{2}(u, v)$ functions as follows

$$
\begin{aligned}
& f_{1}(u, v)=a|u+v|^{2(r+1)}(u+v)+b|u|^{r} u|v|^{r+2}, \\
& f_{2}(u, v)=a|u+v|^{2(r+1)}(u+v)+b|v|^{r} v|u|^{r+2},
\end{aligned}
$$

where $a, b>0$ are constants and $r$ satisfies

$$
\left\{\begin{array}{c}
-1<r \text { if } n=1,2 \\
-1<r \leq \frac{3-n}{n-2} \text { if } n \geq 3
\end{array}\right.
$$

One can easily verify that

$$
u f_{1}(u, v)+v f_{2}(u, v)=2(r+2) F(u, v), \forall(u, v) \in R^{2},
$$

where

$$
F(u, v)=\frac{1}{2(r+2)}\left[a|u+v|^{2(r+2)}+2 b|u v|^{r+2}\right] .
$$

We introduce the energy function associated to problem (1.1) by

$$
\begin{aligned}
E(t)= & \frac{1}{2}\left(\left\|u_{t}\right\|^{2}+\left\|v_{t}\right\|^{2}\right)+\frac{1}{2}\left[\left(\mu_{1} \diamond \nabla u\right)(t)+\left(\mu_{2} \diamond \nabla v\right)(t)\right] \\
& +\frac{1}{2}\left[\left(1-\int_{0}^{t} \mu_{1}(s) d s\right)\|\nabla u(t)\|^{2}+\left(1-\int_{0}^{t} \mu_{2}(s) d s\right)\|\nabla v(t)\|^{2}\right] \\
& +\frac{1}{2(\gamma+1)}\left(\|\nabla u\|^{2(\gamma+1)}+\|\nabla v\|^{2(\gamma+1)}\right)-\int_{\Omega} F(u, v) d x .
\end{aligned}
$$

where $\left(\mu_{i} \diamond \nabla w\right)(t)=\int_{0}^{t} \mu_{i}(t-s)\|\nabla w(t)-\nabla w(s)\|_{2}^{2} d s$.

By computation, we get

$$
\begin{aligned}
E^{\prime}(t)= & \frac{1}{2}\left[\left(\mu_{1}^{\prime} \diamond \nabla u\right)(t)+\left(\mu_{2}^{\prime} \diamond \nabla v\right)(t)\right]-\frac{1}{2}\left(\mu_{1}(t)\|\nabla u\|^{2}+\mu_{2}(t)\|\nabla v\|^{2}\right) \\
& -\int_{\Omega}\left(|u|^{k}+|v|^{l}\right)\left|u_{t}\right|^{p+1} d x-\int_{\Omega}\left(|v|^{\theta}+|u|^{\rho}\right)\left|v_{t}\right|^{q+1} d x .
\end{aligned}
$$




\section{LOCAL EXISTENCE}

In this section, we shall discuss the local existence of a weak solution of problem (1.1). Firstly, we give the definition of weak solutions to problem (1.1).

Definition 1. We say that $(u, v)$ is a weak solution of $(1.1)$ on $[0, T)$ under the assumptions (A1) and (A2) if $u, v \in L^{\infty}\left(0, T ; W_{0}^{1,2(\gamma+1)}(\Omega)\right), u_{t}, v_{t} \in L^{\infty}\left(0, T ; L^{2}(\Omega)\right)$, and satisfies

$$
\begin{aligned}
& \left\langle u^{\prime}(t), \theta\right\rangle-\left\langle u^{1}, \theta\right\rangle+\int_{0}^{t}\left\langle\int_{\Omega} M\left(\|\nabla u\|^{2}\right) \nabla u(\alpha) d \alpha, \nabla \theta\right\rangle d \xi \\
& -\int_{0}^{t}\left\langle\int_{0}^{s} \mu_{1}(\xi-\alpha) \nabla u(\alpha) d \alpha, \nabla \theta\right\rangle d \xi+\int_{0}^{t}\left\langle\left(|u|^{k}+|v|^{l}\right)\left|u^{\prime}(\xi)\right|^{p-1} u^{\prime}(\xi), \theta\right\rangle d \xi \\
& =\int_{0}^{t}\left\langle f_{1}(u(\xi), v(\xi)), \theta\right\rangle d \xi \\
& \left\langle v^{\prime}(t), \phi\right\rangle-\left\langle v^{1}, \phi\right\rangle+\int_{0}^{t}\left\langle\int_{\Omega} M\left(\|\nabla v\|^{2}\right) \nabla v(\alpha) d \alpha, \nabla \phi\right\rangle d \xi \\
& -\int_{0}^{t}\left\langle\int_{0}^{s} \mu_{2}(\xi-\alpha) \nabla v(\alpha) d \alpha, \nabla \phi\right\rangle d \xi+\int_{0}^{t}\left\langle\left(|v|^{\theta}+|u|^{\rho}\right)\left|v^{\prime}(\xi)\right|^{q-1} v^{\prime}(\xi), \phi\right\rangle d \xi \\
& =\int_{0}^{t}\left\langle f_{2}(u(\xi), v(\xi)), \phi\right\rangle d \xi
\end{aligned}
$$

for almost everywhere $t \in[0, T)$ and any test functions $\theta, \phi \in W_{0}^{1,2(\gamma+1)}(\Omega)$.

Theorem 1 (Local existence). Assume assumptions (A1), (A2), (1.6) and $n=$ 1,2,3 hold. Then, for some $T>0$ problem (1.1) has at least a local weak solution $(u, v)$ on $[0, T)$.

Proof. We follow the standard Fadeo-Galerkin approximation to show the existence of solution (1.1). The combination of the Faedo-Galerkin method and the compactness argument give us an efficient method that allows us to deal with some evolution equations with degenerate damping terms.

Let the sequence $\left\{e_{j}: j=1,2, \ldots\right\}$ is an orthogonal basis for $L^{2}(\Omega) \cap W_{0}^{1,2(\gamma+1)}$. Standard results on ordinary differential equations guarantee that there exists only one local solution. We construct approximate solutions $\left(u_{M}(t), v_{M}(t)\right)(M=1,2,3, \ldots)$ in 
the form

$$
u_{M}(t)=\sum_{j=1}^{M} u_{M, j}(t) e_{j}, \quad v_{M}(t)=\sum_{j=1}^{M} v_{M, j}(t) e_{j}
$$

Approximate system

$$
\begin{array}{r}
\left\langle u_{M}^{\prime \prime}(t), e_{j}\right\rangle+\left\langle\int_{\Omega} M\left(\left\|\nabla u_{M}(t)\right\|^{2}\right) \nabla u_{M}(t), \nabla e_{j}\right\rangle-\left\langle\int_{0}^{t} \mu_{1}(t-s) \nabla u_{M}(s) d s, \nabla e_{j}\right\rangle \\
+\left\langle\left(\left|u_{M}(t)\right|^{k}+\left|v_{M}(t)\right|^{l}\right)\left|u_{M}^{\prime}(t)\right|^{p-1} u_{M}^{\prime}(t), e_{j}\right\rangle=\left\langle f_{1}\left(u_{M}(t), v_{M}(t)\right), e_{j}\right\rangle, \quad \text { (2.1) } \\
\left\langle v_{M}^{\prime \prime}(t), e_{j}\right\rangle+\left\langle\int_{\Omega} M\left(\left\|\nabla v_{M}(t)\right\|^{2}\right) \nabla v_{M}(t), \nabla e_{j}\right\rangle-\left\langle\int_{0}^{t} \mu_{2}(t-s) \nabla v_{M}(s) d s, \nabla e_{j}\right\rangle \\
+\left\langle\left(\left|v_{M}(t)\right|^{\theta}+\left|u_{M}(t)\right|^{\rho}\right)\left|v_{M}^{\prime}(t)\right|^{q-1} v_{M}^{\prime}(t), e_{j}\right\rangle=\left\langle f_{2}\left(u_{M}(t), v_{M}(t)\right), e_{j}\right\rangle, \quad \text { (2.2) }
\end{array}
$$

with initial data

$$
\begin{array}{ll}
u_{M}(0)=\sum_{j=1}^{M} u_{M, j}(0) e_{j}, & v_{M}(0)=\sum_{j=1}^{M} v_{M, j}(0) e_{j} \\
u_{M}^{\prime}(0)=\sum_{j=1}^{N} u_{M, j}^{\prime}(0) e_{j}, & v_{M}^{\prime}(0)=\sum_{j=1}^{N} v_{M, j}(0) e_{j}
\end{array}
$$

where

$$
u_{M, j}(0)=\left\langle u^{0}, e_{j}\right\rangle, \quad v_{M, j}(0)=\left\langle v^{0}, e_{j}\right\rangle, u_{M, j}^{\prime}(0)=\left\langle u^{1}, e_{j}\right\rangle, \quad v_{M, j}^{\prime}(0)=\left\langle v^{1}, e_{j}\right\rangle .
$$

\section{A priori estimate}

Multiply (2.1) by $u_{M, j}^{\prime}(t),(2.2)$ by $v_{M, j}^{\prime}(t)$, and summing with respect $j$ from 1 to $M$, we get

$$
\begin{gathered}
\frac{1}{2} \frac{d}{d t}\left[\left\|u_{M}^{\prime}(t)\right\|^{2}+\left(1-\int_{0}^{t} \mu_{1}(s) d s\right)\left\|\nabla u_{M}(t)\right\|^{2}+\left(\mu_{1} \diamond \nabla u_{M}\right)(t)+\frac{1}{\gamma+1}\left\|\nabla u_{M}(t)\right\|^{2(\gamma+1)}\right] \\
+\frac{1}{2} \mu_{1}(t)\left\|\nabla u_{M}(t)\right\|^{2}-\frac{1}{2}\left(\mu_{1}^{\prime} \diamond \nabla u_{M}\right)(t)+\int_{\Omega}\left(\left|u_{M}(t)\right|^{k}+\left|v_{M}(t)\right|^{l}\right)\left|u_{M}^{\prime}(t)\right|^{p+1} d x \\
=\int_{\Omega} f_{1}\left(u_{M}(t), v_{M}(t)\right) u_{M}^{\prime}(t) d x
\end{gathered}
$$

$$
\begin{gathered}
\frac{1}{2} \frac{d}{d t}\left[\left\|v_{M}^{\prime}(t)\right\|^{2}+\left(1-\int_{0}^{t} \mu_{2}(s) d s\right)\left\|\nabla v_{M}(t)\right\|^{2}+\left(\mu_{2} \diamond \nabla v_{M}\right)(t)+\frac{1}{\gamma+1}\left\|\nabla v_{M}(t)\right\|^{2(\gamma+1)}\right] \\
+\frac{1}{2} \mu_{2}(t)\left\|\nabla v_{M}(t)\right\|^{2}-\frac{1}{2}\left(\mu_{2}^{\prime} \diamond \nabla v_{M}\right)(t)+\int_{\Omega}\left(\left|v_{M}(t)\right|^{\theta}+\left|u_{M}(t)\right|^{\rho}\right)\left|v_{M}^{\prime}(t)\right|^{q+1} d x \\
=\int_{\Omega} f_{2}\left(u_{M}(t), v_{M}(t)\right) v_{M}^{\prime}(t) d x
\end{gathered}
$$


Summing (2.5) and (2.6) and integrating from 0 to $t \leq T_{M}$, we obtain

$$
\begin{aligned}
\frac{1}{2} & {\left[\left\|u_{M}^{\prime}(t)\right\|^{2}+\left\|v_{M}^{\prime}(t)\right\|^{2}\right] } \\
& +\frac{1}{2}\left[\left(1-\int_{0}^{t} \mu_{1}(s) d s\right)\left\|\nabla u_{M}(t)\right\|^{2}+\left(1-\int_{0}^{t} \mu_{2}(s) d s\right)\left\|\nabla v_{M}(t)\right\|^{2}\right] \\
& +\frac{1}{2}\left[\left(\mu_{1} \diamond \nabla u_{M}\right)(t)+\left(\mu_{2} \diamond \nabla v_{M}\right)(t)\right] \\
& +\frac{1}{2} \int_{0}^{t}\left[\mu_{1}(s)\left\|\nabla u_{M}(s)\right\|^{2}+\mu_{2}(s)\left\|\nabla v_{M}(s)\right\|^{2}\right] d s \\
& -\frac{1}{2} \int_{0}^{t}\left[\left(\mu_{1}^{\prime} \diamond \nabla u_{M}\right)(s)+\left(\mu_{2}^{\prime} \diamond \nabla v_{M}\right)(s)\right] d s \\
& +\frac{1}{2(\gamma+1)}\left[\left\|\nabla u_{M}(t)\right\|^{2(\gamma+1)}+\left\|\nabla v_{M}(t)\right\|^{2(\gamma+1)}\right] \\
& +\int_{0}^{t} \int_{\Omega}\left(\left|u_{M}(s)\right|^{k}+\left|v_{M}(s)\right|^{l}\right)\left|u_{M}^{\prime}(s)\right|^{p+1} d x d s \\
& +\int_{0}^{t} \int_{\Omega}\left(\left|v_{M}(s)\right|^{\theta}+\left|u_{M}(s)\right|^{\rho}\right)\left|v_{M}^{\prime}(s)\right|^{q+1} d x d s \\
= & \frac{1}{2}\left(\left\|u_{M}^{\prime}(0)\right\|^{2}+\left\|v_{M}^{\prime}(0)\right\|^{2}+\left\|\nabla u_{M}(0)\right\|^{2}+\left\|\nabla v_{M}(0)\right\|^{2}\right) \\
& +\frac{1}{2(\gamma+1)}\left(\left\|\nabla u_{M}(0)\right\|^{2(\gamma+1)}+\left\|\nabla v_{M}(0)\right\|^{2(\gamma+1)}\right) \\
& +\int_{0}^{t} \int_{\Omega}\left[f_{1}\left(u_{M}(s), v_{M}(s)\right) u_{M}^{\prime}(s)+f_{2}\left(u_{M}(s), v_{M}(s)\right) v_{M}^{\prime}(s)\right] d x d s \\
\leq & C_{0}+\int_{0}^{t} \int_{\Omega}\left[f_{1}\left(u_{M}(s), v_{M}(s)\right) u_{M}^{\prime}(s)+f_{2}\left(u_{M}(s), v_{M}(s)\right) v_{M}^{\prime}(s)\right] d x d s
\end{aligned}
$$

with a positive constant

$$
C_{0}=C\left(\left|u^{0}\right|_{H^{1}(\Omega)},\left|v^{0}\right|_{H^{1}(\Omega)},\left|u^{1}\right|_{L^{2}(\Omega)},\left|v^{1}\right|_{L^{2}(\Omega)},\left|u^{0}\right|_{W^{1,2(\gamma+1)}(\Omega)},\left|v^{0}\right|_{W^{1,2(\gamma+1)}(\Omega)}\right) .
$$

To estimate the last term in (2.7) applying (1.5) and using Young inequalities, Hölder inequalities and Sobolev embedding theorem, we have

$$
\begin{aligned}
& \left|\int_{\Omega} f_{1}\left(u_{M}, v_{M}\right) u_{M}^{\prime} d x\right| \leq C \int_{\Omega}\left(\left|u_{M}+v_{M}\right|^{2 r+3}\left|u_{M}^{\prime}\right|+\left|v_{M}\right|^{r+2}\left|u_{M}\right|^{r+1}\left|u_{M}^{\prime}\right|\right) d x \\
& \leq C\left[\left(\left\|u_{M}\right\|_{2(2 r+3)}^{2 r+3}+\left\|v_{M}\right\|_{2(2 r+3)}^{2 r+3}\right)\left\|u_{M}^{\prime}\right\|+\left\|u_{M}\right\|_{4(r+1)}^{r+1}\left\|v_{M}\right\|_{4(r+2)}^{r+2}\left\|u_{M}^{\prime}\right\|\right] \\
& \leq C\left[\left\|\nabla u_{M}\right\|^{2(2 r+3)}+\left\|\nabla v_{M}\right\|^{2(2 r+3)}+\left\|\nabla u_{M}\right\|^{2(r+1)}\left\|\nabla v_{M}\right\|^{2(r+2)}+\left\|u_{M}^{\prime}\right\|^{2}\right] .
\end{aligned}
$$


In the same way, we obtain

$$
\begin{aligned}
\left|\int_{\Omega} f_{2}\left(u_{M}, v_{M}\right) v_{N}^{\prime} d x\right| \leq & C\left[\left\|\nabla u_{M}\right\|^{2(2 r+3)}+\left\|\nabla v_{M}\right\|^{2(2 r+3)}\right. \\
& \left.+\left\|\nabla u_{M}\right\|^{2(r+2)}\left\|\nabla v_{M}\right\|^{2(r+1)}+\left\|v_{M}^{\prime}\right\|^{2}\right] .
\end{aligned}
$$

Now, by putting

$$
\begin{aligned}
y_{M}(t):= & \left\|u_{M}^{\prime}(t)\right\|^{2}+\left\|v_{M}^{\prime}(t)\right\|^{2}+\left\|\nabla u_{M}(t)\right\|^{2}+\left\|\nabla v_{M}(t)\right\|^{2} \\
& +\frac{1}{l(\gamma+1)}\left[\left\|\nabla u_{M}(t)\right\|^{2(\gamma+1)}+\left\|\nabla v_{M}(t)\right\|^{2(\gamma+1)}\right],
\end{aligned}
$$

where $l=\min \left\{l_{1}, l_{2}\right\}<1$. Then, we infer from (2.7)-(2.9)

$$
\begin{aligned}
y_{M}( & (t)+\frac{1}{l}\left[\left(\mu_{1} \diamond \nabla u_{M}\right)(t)+\left(\mu_{2} \diamond \nabla v_{M}\right)(t)\right] \\
& +\frac{1}{l} \int_{0}^{t}\left[\mu_{1}(s)\left\|\nabla u_{M}(s)\right\|^{2}+\mu_{2}(s)\left\|\nabla v_{M}(s)\right\|^{2}\right] d s \\
& -\frac{1}{l} \int_{0}^{t}\left[\left(\mu_{1}^{\prime} \diamond \nabla u_{M}\right)(s)+\left(\mu_{2}^{\prime} \diamond \nabla v_{M}\right)(s)\right] d s \\
& +\frac{2}{l} \int_{0}^{t} \int_{\Omega}\left(\left|u_{M}(s)\right|^{k}+\left|v_{M}(s)\right|^{l}\right)\left|u_{M}^{\prime}(s)\right|^{p+1} d x d s \\
& +\frac{2}{l} \int_{0}^{t} \int_{\Omega}\left(\left|v_{M}(s)\right|^{\theta}+\left|u_{M}(s)\right|^{\rho}\right)\left|v_{M}^{\prime}(s)\right|^{q+1} d x d s \leq C_{0}+C \int_{0}^{t} y_{M}^{(2 r+3)}(s) d s,
\end{aligned}
$$

Particularly, $y_{M}(t)$ satisfies the inequality

$$
y_{M}(t) \leq C_{0}+C \int_{0}^{t} y_{M}^{(2 r+3)}(s) d s .
$$

By applying Grönwall inequality we have

$$
y_{M}(t) \leq C_{1} \text { for all } t \in[0, T] .
$$

The estimates follow from (2.10) and (2.12):

$u_{M}, v_{M}$ are uniformly bounded in $L^{\infty}\left(0, T ; W_{0}^{2(\gamma+1)}(\Omega)\right)$;

$u_{M}^{\prime}, v_{M}^{\prime}$ are uniformly bounded in $L^{\infty}\left(0, T ; L^{2}(\Omega)\right)$;

The sequences $\left\{\int_{0}^{t} \int_{\Omega}\left(\left|u_{M}(s)\right|^{k}+\left|v_{M}(s)\right|^{l}\right)\left|u_{M}^{\prime}(s)\right|^{p+1} d x d s\right\}$ and $\left\{\int_{0}^{t} \int_{\Omega}\left(\left|v_{M}(s)\right|^{\theta}+\left|u_{M}(s)\right|^{\rho}\right)\left|v_{M}^{\prime}(s)\right|^{q+1} d x d s\right\}$ are uniformly bounded in $L^{\infty}(0, T)$. Then

$$
\begin{aligned}
& u_{M} \rightarrow u, \quad v_{M} \rightarrow v \quad \text { weakly } * \text { in } L^{\infty}\left(0, T, W_{0}^{2(\gamma+1)}(\Omega)\right), \\
& u_{M}^{\prime} \rightarrow u^{\prime}, \quad v_{M}^{\prime} \rightarrow v^{\prime} \quad \text { weakly } * \text { in } L^{\infty}\left(0, T, L^{2}(\Omega)\right),
\end{aligned}
$$


By applying the techniques in [10], we obtain the sequence of approximate solutions $\left(u_{M}, v_{M}\right)$ satisfying

$$
\left\{\begin{array}{c}
\left\{u_{M}\right\},\left\{v_{M}\right\} \text { are Cauchy sequences in } L^{\infty}\left(0, T, W_{0}^{2(\gamma+1)}(\Omega)\right), \\
\left\{u_{M}^{\prime}\right\},\left\{v_{M}^{\prime}\right\} \text { are Cauchy sequences in } L^{\infty}\left(0, T, L^{2}(\Omega)\right) .
\end{array}\right.
$$

\section{Limiting process}

Integrating (2.1) and (2.2) over $[0, T]$, we get

$$
\begin{gathered}
\left\langle u_{M}^{\prime}(t), e_{j}\right\rangle-\left\langle u_{M}^{\prime}(0), e_{j}\right\rangle+\int_{0}^{T}\left\langle M\left(\left\|\nabla u_{M}(s)\right\|^{2}\right) \nabla u_{M}(s), \nabla e_{j}\right\rangle d s \\
-\int_{0}^{T}\left\langle\int_{0}^{s} \mu_{1}(s-\tau) \nabla u_{M}(\tau) d \tau, \nabla e_{j}\right\rangle d s+\int_{0}^{T}\left\langle\left(\left|u_{M}\right|^{k}+\left|v_{M}\right|^{l}\right)\left|u_{M}^{\prime}\right|^{p-1} u_{M}^{\prime}, e_{j}\right\rangle d s \\
=\int_{0}^{T}\left\langle f_{1}\left(u_{M}(s), v_{M}(s)\right), e_{j}\right\rangle d s, \quad \text { (2.13) } \\
\left\langle v_{M}^{\prime}(t), e_{j}\right\rangle-\left\langle v_{M}^{\prime}(0), e_{j}\right\rangle+\int_{0}^{T}\left\langle M\left(\left\|\nabla v_{M}(s)\right\|^{2}\right) \nabla v_{M}(s), \nabla e_{j}\right\rangle d s \\
-\int_{0}^{T}\left\langle\int_{0}^{s} \mu_{2}(s-\tau) \nabla v_{M}(\tau) d \tau, \nabla e_{j}\right\rangle d s+\int_{0}^{T}\left\langle\left(\left|v_{M}\right|^{\theta}+\left|u_{M}\right|^{\rho}\right)\left|v_{M}^{\prime}\right|^{q-1} v_{M}^{\prime}, e_{j}\right\rangle d s \\
=\int_{0}^{T}\left\langle f_{2}\left(u_{M}(s), v_{M}(s)\right), e_{j}\right\rangle d s .
\end{gathered}
$$

Now, we can pass to the limit in (2.13) and (2.14) as $M \rightarrow \infty$. Therefore, this completes our proof of the local existence of a weak solution.

\section{BLOW UP SOLUTIONS}

Our main result in this section is to show the blow up result of the solution to (1.1). For this purpose, we need the following lemmas.

Lemma 1 ([6]). There exist two positive constants $c_{0}$ and $c_{1}$ such that

$$
c_{0}\left(|u|^{2(r+2)}+|v|^{2(r+2)}\right) \leq 2(r+2) F(u, v) \leq c_{1}\left(|u|^{2(r+2)}+|v|^{2(r+2)}\right)
$$

is satisfied.

Lemma 2. Suppose that (1.6) holds. Then there exists $\eta>0$ such that for the solution $(u, v)$

$$
\begin{aligned}
& \|u+v\|_{2(r+2)}^{2(r+2)}+2\|u v\|_{r+2}^{r+2} \\
& \quad \leq \eta\left(\frac{1}{\gamma+1}\left(\|\nabla u\|^{2(\gamma+1)}+\|\nabla v\|^{2(\gamma+1)}\right)+l_{1}\|\nabla u\|^{2}+l_{2}\|\nabla v\|^{2}\right)^{r+2} .
\end{aligned}
$$


Proof. By applying Minkowski inequality, we get

$$
\|u+v\|_{2(r+2)}^{2} \leq 2\left(\|u\|_{2(r+2)}^{2}+\|v\|_{2(r+2)}^{2}\right) .
$$

Moreover, applying Hölder's inequality and Young's inequality we have

$$
\|u v\|_{(r+2)} \leq\|u\|_{2(r+2)}\|v\|_{2(r+2)} \leq \frac{1}{2}\left(\|u\|_{2(r+2)}^{2}+\|v\|_{2(r+2)}^{2}\right) .
$$

A combination of (3.3), (3.4), and the embedding $H_{0}^{1}(\Omega) \hookrightarrow L^{2(r+2)}(\Omega)$, imply

$$
\begin{gathered}
\|u+v\|_{2(r+2)}^{2} \leq C\left(l_{1}\|\nabla u\|^{2}+l_{2}\|\nabla v\|^{2}\right), \\
\|u v\|_{(r+2)} \leq C\left(l_{1}\|\nabla u\|^{2}+l_{2}\|\nabla v\|^{2}\right) .
\end{gathered}
$$

Then, we have

$$
\|\nabla u\|^{2}+\|\nabla v\|^{2} \leq\|\nabla u\|^{2(\gamma+1)}+\|\nabla v\|^{2(\gamma+1)} .
$$

Thus,

$$
\|\nabla u\|^{2(\gamma+1)}+\|\nabla v\|^{2(\gamma+1)} \geq l_{1}\|\nabla u\|^{2}+l_{2}\|\nabla v\|^{2} .
$$

Combining (3.3), (3.4) and (3.8), we have

$$
\begin{gathered}
\|u+v\|_{2(r+2)}^{2} \leq C\left[\frac{1}{\gamma+1}\left(\|\nabla u\|^{2(\gamma+1)}+\|\nabla v\|^{2(\gamma+1)}\right)+l_{1}\|\nabla u\|^{2}+l_{2}\|\nabla v\|^{2}\right] \\
\|u v\|_{r+2} \leq C\left[\frac{1}{\gamma+1}\left(\|\nabla u\|^{2(\gamma+1)}+\|\nabla v\|^{2(\gamma+1)}\right)+l_{1}\|\nabla u\|^{2}+l_{2}\|\nabla v\|^{2}\right]
\end{gathered}
$$

Thus, the lemma follows.

We take $a=b=1$ for simplicity and introduce the following:

$$
B=\eta^{\frac{1}{2(r+2)}}, \alpha_{1}=B^{-\frac{r+2}{r+1}}, E_{1}=\left(\frac{1}{2}-\frac{1}{2(r+2)}\right) \alpha_{1}^{2}, E_{2}=\left(\frac{1}{2(\gamma+1)}-\frac{1}{2(r+2)}\right) \alpha_{1}^{2},
$$

where $\eta$ is the optimal constant in (3.2).

Let

$$
\Gamma(t):=\left(1-\int_{0}^{t} \mu_{1}(s) d s\right)\|\nabla u\|^{2}+\left(1-\int_{0}^{t} \mu_{2}(s) d s\right)\|\nabla v\|^{2}+\left(\mu_{1} \diamond \nabla u\right)+\left(\mu_{2} \diamond \nabla v\right) .
$$

The next lemma will act an important role in our proof, and it is similar to a lemma introduced firstly by Vitillaro [16].

Lemma 3. Suppose that assumptions (A1) and (1.6) hold. Let $(u, v)$ be a solution of (1.1). Moreover, assume that $E(0)<E_{1}$ and

$$
\left(\frac{1}{\gamma+1}\left(\|\nabla u(0)\|^{2(\gamma+1)}+\|\nabla v(0)\|^{2(\gamma+1)}\right)+\Gamma(0)\right)^{\frac{1}{2}}>\alpha_{1} .
$$


Then there exists a constant $\alpha_{2}>\alpha_{1}$ such that

$$
\begin{gathered}
\left(\frac{1}{\gamma+1}\left(\|\nabla u\|^{2(\gamma+1)}+\|\nabla v\|^{2(\gamma+1)}\right)+\Gamma(t)\right)^{\frac{1}{2}} \geq \alpha_{2}, \text { for } t>0 \\
\left(\|u+v\|_{2(r+2)}^{2(r+2)}+2\|u v\|_{r+2}^{r+2}\right)^{\frac{1}{2(r+2)}} \geq B \alpha_{2}, \text { for } t>0 .
\end{gathered}
$$

for all $t \in[0, T)$.

Proof. We first note that by (1.9), (3.2), (3.12) and the definition of $B$, we have

$$
\begin{aligned}
E(t) \geq & \frac{1}{2(\gamma+1)}\left(\|\nabla u\|^{2(\gamma+1)}+\|\nabla v\|^{2(\gamma+1)}\right)+\frac{1}{2} \Gamma(t)-\int_{\Omega} F(u, v) d x \\
= & \frac{1}{2(\gamma+1)}\left(\|\nabla u\|^{2(\gamma+1)}+\|\nabla v\|^{2(\gamma+1)}\right)+\frac{1}{2} \Gamma(t) \\
& -\frac{1}{2(r+2)}\left(\|u+v\|_{2(r+2)}^{2(r+2)}+2\|u v\|_{r+2}^{r+2}\right) \\
\geq & \frac{1}{2(\gamma+1)}\left(\|\nabla u\|^{2(\gamma+1)}+\|\nabla v\|^{2(\gamma+1)}\right)+\frac{1}{2} \Gamma(t) \\
& -\frac{\eta}{2(r+2)}\left[\frac{1}{\gamma+1}\left(\|\nabla u\|^{2(\gamma+1)}+\|\nabla v\|^{2(\gamma+1)}\right)+l_{1}\|\nabla u\|^{2}+l_{2}\|\nabla v\|^{2}\right]^{r+2} \\
\geq & \frac{1}{2}\left[\frac{1}{\gamma+1}\left(\|\nabla u\|^{2(\gamma+1)}+\|\nabla v\|^{2(\gamma+1)}\right)+\Gamma(t)\right] \\
& -\frac{B^{2(r+2)}}{2(r+2)}\left[\frac{1}{\gamma+1}\left(\|\nabla u\|^{2(\gamma+1)}+\|\nabla v\|^{2(\gamma+1)}\right)+\Gamma(t)\right]^{r+2} \\
= & \frac{1}{2} \alpha^{2}-\frac{B^{2(r+2)}}{2(r+2)} \alpha^{2(r+2)}=G(\alpha),
\end{aligned}
$$

where

$$
\alpha=\left[\frac{1}{\gamma+1}\left(\|\nabla u\|^{2(\gamma+1)}+\|\nabla v\|^{2(\gamma+1)}\right)+\Gamma(t)\right]^{1 / 2} .
$$

It is not difficult to verify that $G$ is increasing for $0<\alpha<\alpha_{1}$, decreasing for $\alpha>\alpha_{1}$, $G(\alpha) \rightarrow-\infty$ as $\alpha \rightarrow \infty$, and

$$
G\left(\alpha_{1}\right)=\frac{1}{2} \alpha_{1}^{2}-\frac{B^{2(r+2)}}{2(r+2)} \alpha_{1}^{2(r+2)}=E_{1},
$$

where $\alpha_{1}$ is given in (3.11). Since $E(0)<E_{1}$, there exists $\alpha_{2}>\alpha_{1}$ such that $G\left(\alpha_{2}\right)=E(0)$.

Set $\alpha_{0}=\left[\frac{1}{\gamma+1}\left(\|\nabla u(0)\|^{2(\gamma+1)}+\|\nabla v(0)\|^{2(\gamma+1)}\right)+\Gamma(0)\right]^{1 / 2}$. Then by (3.16) we get $G\left(\alpha_{0}\right) \leq E(0)=G\left(\alpha_{2}\right)$, which implies that $\alpha_{0} \geq \alpha_{2}$. 
Now, to establish (3.14), we suppose by contradiction that

$$
\left[\frac{1}{\gamma+1}\left(\left\|\nabla u\left(t_{0}\right)\right\|^{2(\gamma+1)}+\left\|\nabla v\left(t_{0}\right)\right\|^{2(\gamma+1)}\right)+\Gamma\left(t_{0}\right)\right]^{1 / 2}<\alpha_{2},
$$

for some $t_{0}>0$. By the continuity of $\left[\frac{1}{\gamma+1}\left(\left\|\nabla u\left(t_{0}\right)\right\|^{2(\gamma+1)}+\left\|\nabla v\left(t_{0}\right)\right\|^{2(\gamma+1)}\right)+\Gamma\left(t_{0}\right)\right]$, we can choose $t_{0}$ such that

$$
\left[\frac{1}{\gamma+1}\left(\left\|\nabla u\left(t_{0}\right)\right\|^{2(\gamma+1)}+\left\|\nabla v\left(t_{0}\right)\right\|^{2(\gamma+1)}\right)+\Gamma\left(t_{0}\right)\right]^{1 / 2}>\alpha_{1} .
$$

Again, using (3.16) we get

$$
E\left(t_{0}\right) \geq G\left(\left[\frac{1}{\gamma+1}\left(\left\|\nabla u\left(t_{0}\right)\right\|^{2(\gamma+1)}+\left\|\nabla v\left(t_{0}\right)\right\|^{2(\gamma+1)}\right)+\Gamma\left(t_{0}\right)\right]^{1 / 2}\right)>G\left(\alpha_{2}\right)=E(0) .
$$

This is impossible since $E(t) \leq E(0)$ for all $t \in[0, T)$. Hence (3.14) is established.

To prove (3.15), we apply (1.9) to get

$$
\begin{aligned}
\frac{1}{2(\gamma+1)}\left(\|\nabla u\|^{2(\gamma+1)}+\|\nabla v\|^{2(\gamma+1)}\right. & +\frac{1}{2} \Gamma(t) \\
\leq & E(0)+\frac{1}{2(r+2)}\left(\|u+v\|_{2(r+2)}^{2(r+2)}+2\|u v\|_{r+2}^{r+2}\right) .
\end{aligned}
$$

Consequently, (3.14) yields

$$
\begin{aligned}
& \frac{1}{2(r+2)}\left(\|u+v\|_{2(r+2)}^{2(r+2)}+2\|u v\|_{r+2}^{r+2}\right) \\
& \geq \frac{1}{2(\gamma+1)}\left(\|\nabla u\|^{2(\gamma+1)}+\|\nabla v\|^{2(\gamma+1)}\right)+\frac{1}{2} \Gamma(t)-E(0) \\
& \quad \geq \frac{1}{2} \alpha_{2}^{2}-G\left(\alpha_{2}\right)=\frac{B^{2(r+2)}}{2(r+2)} \alpha_{2}^{2(r+2)}
\end{aligned}
$$

Therefore, (3.18) and (3.11) yield the desired result.

Theorem 2. Assume that (A1), (A2) and (1.6) hold. Assume further that

$$
2(r+2)>\max \{2(\gamma+1), k+p+1, l+p+1, \theta+q+1, \rho+q+1\} .
$$

Then any the solution of the problem (1.1) with initial data satisfying

$$
\left[\frac{1}{\gamma+1}\left(\|\nabla u(0)\|^{2(\gamma+1)}+\|\nabla v(0)\|^{2(\gamma+1)}\right)+\Gamma(0)\right]^{\frac{1}{2}}>\alpha_{1}, E(0)<E_{2},
$$

cannot exist for all time, where $\alpha_{1}$ and $E_{2}$ are defined in (3.11). 
Proof. We suppose that the solution exists for all time and we reach to a contradiction. Set

$$
H(t)=E_{2}-E(t) .
$$

By applying (1.9) and (3.19), we have

$$
\begin{aligned}
0<H(0) \leq H(t)= & E_{2}-\frac{1}{2}\left(\left\|u_{t}\right\|_{2}^{2}+\left\|v_{t}\right\|_{2}^{2}\right)-\frac{1}{2} \Gamma(t) \\
& -\frac{1}{2(\gamma+1)}\left(\|\nabla u\|^{2(\gamma+1)}+\|\nabla v\|^{2(\gamma+1)}\right)+\int_{\Omega} F(u, v) d x .
\end{aligned}
$$

From (3.18) and (3.1), we have

$$
\begin{aligned}
E_{2} & -\frac{1}{2}\left(\left\|u_{t}\right\|_{2}^{2}+\left\|v_{t}\right\|_{2}^{2}\right)-\frac{1}{2} \Gamma(t) \\
& -\frac{1}{2(\gamma+1)}\left(\|\nabla u\|^{2(\gamma+1)}+\|\nabla v\|^{2(\gamma+1)}\right)+\int_{\Omega} F(u, v) d x \\
\leq & E_{1}-\frac{1}{2} \alpha_{1}^{2}+\frac{c_{1}}{2(r+2)}\left(\|u\|_{2(r+2)}^{2(r+2)}+\|v\|_{2(r+2)}^{2(r+2)}\right) \\
\leq & -\frac{1}{2(r+2)} \alpha_{1}^{2}+\frac{c_{1}}{2(r+2)}\left(\|u\|_{2(r+2)}^{2(r+2)}+\|v\|_{2(r+2)}^{2(r+2)}\right) \\
\leq & \frac{c_{1}}{2(r+2)}\left(\|u\|_{2(r+2)}^{2(r+2)}+\|v\|_{2(r+2)}^{2(r+2)}\right) .
\end{aligned}
$$

By combining (3.20) and (3.21), we have

$$
0<H(0) \leq H(t) \leq \frac{c_{1}}{2(r+2)}\left(\|u\|_{2(r+2)}^{2(r+2)}+\|v\|_{2(r+2)}^{2(r+2)}\right) .
$$

We then define with $\varepsilon>0$

$$
\Psi(t)=H^{1-\sigma}(t)+\varepsilon\left(\int_{\Omega} u_{t} u d x+\int_{\Omega} v_{t} v d x\right) .
$$

The remainder of the proof is similar to the proof of Theorem 12 in [9], and then we get the desired result.

\section{ACKNOWLEDGEMENT}

The authors would like to thank the referees for the careful reading and suggestions that improved this manuscript.

\section{REFERENCES}

[1] A. Benaissa, D. Ouchenane, and K. Zennir, "Blow up of positive initial-energy solutions to systems of nonlinear wave equations with degenerate damping and source terms," Nonlinear Studies. The International Journal, vol. 19, no. 4, pp. 523-535, 2012.

[2] B. Feng, Y. Qin, and M. Zhang, "General decay for a system of nonlinear viscoelastic wave equations with weak damping," Boundary Value Problems, vol. 2012, no. 1, p. 146, 2012, doi: 10.1186/1687-2770-2012-146. 
[3] X. Han and M. Wang, "Global existence and blow-up of solutions for a system of nonlinear viscoelastic wave equations with damping and source," Nonlinear Analysis: Theory, Methods \& Applications, vol. 71, no. 11, pp. 5427-5450, 2009, doi: 10.1016/j.na.2009.04.031.

[4] V. Ho, N. V. Huynh, and P. Le, "Solutions of homogeneous fractional p-Kirchhoff equations in $R^{n}$," Miskolc Mathematical Notes, vol. 20, no. 2, pp. 957-968, 2019, doi: 10.18514/MMN.2019.2869.

[5] W. Liu, G. Li, and L. Hong, "General decay and blow-up of solutions for a system of viscoelastic equations of Kirchhoff type with strong damping," Journal of Function Spaces, vol. 2014, 2014, doi: 10.1155/2014/284809.

[6] S. A. Messaoudi and B. Said-Houari, "Global nonexistence of positive initial-energy solutions of a system of nonlinear viscoelastic wave equations with damping and source terms," Journal of Mathematical Analysis and Applications, vol. 365, no. 1, pp. 277-287, 2010, doi: 10.1016/j.jmaa.2009.10.050.

[7] E. Pişkin, "Global nonexistence of solutions for a system of viscoelastic wave equations with weak damping terms," Malaya Journal of Matematik, vol. 3, no. 2, pp. 168-174, 2015.

[8] E. Pişkin, "A lower bound for the blow up time of a system of viscoelastic wave equations with nonlinear damping and source terms," Journal of Nonlinear Functional Analysis, vol. 2017, pp. 1-9, 2017, doi: 10.23952/jnfa.2017.3.

[9] E. Pişkin and F. Ekinci, "General decay and blowup of solutions for coupled viscoelastic equation of Kirchhoff type with degenerate damping terms," Mathematical Methods in the Applied Sciences, vol. 42, no. 16, pp. 5468-5488, 2019, doi: 10.1002/mma.5429.

[10] E. Pişkin, F. Ekinci, and K. Zennir, "Local existence and blow-up of solutions for coupled viscoelastic wave equations with degenerate damping terms," Theoretical and Applied Mechanics, no. 00, pp. 8-8, 2020, doi: 10.2298/TAM200428008P.

[11] M. A. Ragusa and A. Tachikawa, "Partial regularity of the minimizers of quadratic functionals with vmo coefficients," Journal of the London Mathematical Society, vol. 72, no. 3, pp. 609-620, 2005, doi: 10.1112/S002461070500699X.

[12] M. A. Ragusa and A. Tachikawa, "Regularity for minimizers for functionals of double phase with variable exponents," Advances in Nonlinear Analysis, vol. 9, no. 1, pp. 710-728, 2019, doi: 10.1515/anona-2020-0022.

[13] M. A. Rammaha and S. Sakuntasathien, "Global existence and blow up of solutions to systems of nonlinear wave equations with degenerate damping and source terms," Nonlinear Analysis: Theory, Methods \& Applications, vol. 72, no. 5, pp. 2658-2683, 2010, doi: 10.1016/j.na.2009.11.013.

[14] B. Said-Houari, S. A. Messaoudi, and A. Guesmia, "General decay of solutions of a nonlinear system of viscoelastic wave equations," Nonlinear Differential Equations and Applications NoDEA, vol. 18, no. 6, pp. 659-684, 2011, doi: 10.1007/s00030-011-0112-7.

[15] M. R. H. Tavani and M. Khodabakhshi, "Existence of weak solutions for three-point boundary value problems of Kirchhoff-type," Miskolc Mathematical Notes, vol. 20, no. 1, pp. 539-554, 2019, doi: 10.18514/MMN.2019.2868.

[16] E. Vitillaro, "Global nonexistence theorems for a class of evolution equations with dissipation," Archive for Rational Mechanics and Analysis, vol. 149, no. 2, pp. 155-182, 1999.

[17] S.-T. Wu, "General decay of solutions for a nonlinear system of viscoelastic wave equations with degenerate damping and source terms," Journal of Mathematical Analysis and Applications, vol. 406, no. 1, pp. 34-48, 2013, doi: 10.1016/j.jmaa.2013.04.029.

[18] K. Zennir, "Growth of solutions to system of nonlinear wave equations with degenerate damping and strong sources," Nonlinear Anal Appl, vol. 2013, pp. 1-11, 2013, doi: 10.5899/2013/jnaa00210 . 
Authors' addresses

\section{Erhan Pişkin}

Dicle University, Department of Mathematics, 21280 Diyarbakir, Turkey

E-mail address: episkinedicle.edu.tr

\section{Fatma Ekinci}

(Corresponding author) Dicle University, Department of Mathematics, 21280 Diyarbakir, Turkey

E-mail address: ekincifatma2017@gmail.com 